\title{
Impact of Molten Pool Dynamics on Resultant Surface Structures During Direct Laser Interference Patterning
}

\author{
Bogdan Voisiat $^{*}{ }^{1}$, Stephan Milles ${ }^{1}$, and Andrés Fabian Lasagni ${ }^{1,2}$ \\ ${ }^{1}$ Institut für Fertigungstechnik, Technische Universität Dresden, George-Baehr-Str. 3c, 01069 \\ Dresden, Germany \\ ${ }^{2}$ Fraunhofer Institut für Werkstoff und Strahltechnik IWS, Winterbergstr. 28, 01277 Dresden, \\ Germany \\ *Corresponding author's e-mail: bogdan.voisiat@tu-dresden.de
}

\begin{abstract}
Direct laser interference patterning (DLIP) is a well-established technology, which is often used for material surface structuring when both high-throughput and resolution are needed. In this technique, the periodical features of the surface structures are produced simultaneously within a single laser spot. This requires taking into consideration the interaction between the ablation processes of each feature, which includes heat transfer and the interaction between the molten material. In this study, we demonstrate how the molten material dynamics during the four-beam picosecond DLIP of the steel surface affect the topography of the fabricated structure. The detailed analysis of the structures produced with various laser fluences reveals three distinct mechanisms of structure formation, which lead to the structure consisting not only of periodically distributed craters but also of pillars, formed due to the molten material flow dynamics. The topography of the structures formed with different laser fluences and the number of laser pulses is investigated using confocal and scanning electron microscopy techniques.
\end{abstract}

DOI: $10.2961 /$ jlmn.2021.01.2006

Keywords: direct laser interference patterning, melt pool dynamics, periodic surface structures, stainless steel, pillar-like structure.

\section{Introduction}

Direct laser interference patterning (DLIP) is a well-established technology used for material surface structuring. It implements the interference patterns produced by overlapping split laser beams on a substrate-surface to melt or ablate the material locally at the interference maxima positions [1]. DLIP is often applied when submicrometer periodical structures are needed to be fabricated on the relatively big surface areas [2], which are used to add or enhance different functionalities such as superhydrophobicity [3], ice-repellency [4], antibacterial property [5], altered magnetic properties [6] and diffractive color effects [7].

Depending on the number of interfering beams, different types of periodical surface structures can be fabricated, namely line-like (two beams), dot-like with triangular (three beams) or square-like (four beams) geometries, as well as hexagonal symmetries (six beams) [1]. In all cases, the periodical features (trenches and pits) of the structures are produced simultaneously with the single laser spot. This characteristic is different from conventional laser-based fabrication methods (e.g. direct laser writing), in which laser ablation occurs in a single focused laser beam (typically 10-30 $\mu \mathrm{m}$ spot size), and each feature is formed successively with each forthcoming laser pulse. This difference substantially changes the circumstances of the structure formation mechanism, since the simultaneous formation of the structures' features in DLIP requires to consider the interaction between the ablated features. This coupling includes heat transfer as well as the interaction between the molten material that governs the final topography of the fabricated structure.
The heat transfer effect between interference peaks on aluminum structuring with ns laser pulses was investigated by M. D'Alessandria et al. [8]. They showed that the higher structure depths could be achieved for larger periods. When the period is small and becomes close to the thermal diffusion length, the temperature difference between maxima and minima is low and does not lead to local and periodic melting of the surface, leading to shallow structure depths. Gedvilas et al. introduced a temperature modulation depth (M) as a parameter to evaluate the interference ablation quality, which was divided into three categories: ultra-fine ablation $(M>0.96)$ ablation and melting, $(0.96>M>0.4)$, melting $(\mathrm{M}<0.4)$ [9].

When a metal is ablated at the interference maxima, also a part is melted, which later solidifies. Before solidification, the molten material experiences displacement due to the Marangoni convection and/or recoil pressure $[10,11]$. Nakata et al. showed that due to the liquid material flow dynamics during the femtosecond DLIP of thin gold film, complex shapes (in the form free-standing gold nanowhisker) of the periodic features can be formed [12]. At higher laser fluences, more material can be molten, as well as stronger melt pool displacement occurs, causing a complex interaction of the liquid coming from neighboring interference intensity peaks. Such phenomena can increase the depth of the formed periodic line-like structure, as the molten material is collected on top of the non-ablated areas [13] or form pillars when an inverted three-beam interference intensity profile is used [14]. 
In this research, we present a detailed analysis of the periodical structure formation mechanism during the fourbeam picosecond DLIP of stainless steel. The topography of the structures formed with different laser fluences and the number of laser pulses is investigated using confocal microscopy. The scanning electron microscopy (SEM) was also implemented to show a more detailed view of the structure morphology. The results showed a high impact of melted material flow dynamics on the shape of the fabricated structure.

\section{Experiments and methods}

\subsection{Materials}

The laser processing experiments were performed on $0.8 \mathrm{~mm}$ thick metal plates made of $\mathrm{X} 6 \mathrm{Cr} 17$ corrosion resisting ferritic steel (1.4016). The surface of the plates was electro-polished, providing a surface roughness $\mathrm{Ra}$ of $60 \mathrm{~nm}$. Before the laser process, the substrates were cleaned from contaminations using isopropanol.

\subsection{Experimental setup}

The structuring of the steel (1.4016) samples was performed using four-beam DLIP. A picosecond (70 ps) solidstate pulsed laser (neoMos 70ps, NeoLASE GmbH, Hannover, Germany) with a wavelength of $532 \mathrm{~nm}$ (frequencydoubled) and a repetition rate of $10 \mathrm{kHz}$ was used. To obtain the four-beam interference pattern, an optical setup containing a diffractive optical element (DOE), a pyramid (made of fused silica), and a focusing lens was used. In this way, the primary laser beam is split into four sub-beams by the DOE and then parallelized with the optical pyramid. The four parallelly propagating beams are then collected and focused on a single position on the samples' surface by a focusing lens, as it is shown in Fig. 1a.

The intensity profile that is formed by interference of four beams contains round-shaped peaks distributed periodically in a square lattice (see Fig. 1b). The intensity of the peaks reduces from the center of interference spot following a Gaussian distribution. The more detailed description of the four beams interference profile can be found here $[15,16]$.

The spatial period of the four-beam interference pattern is directly proportional to the laser wavelength $(\lambda)$ and inversely proportional to the sine of the interference angle $(\theta)$ :

$$
\Lambda_{4}=\frac{\lambda}{\sqrt{2} \sin (\theta)} .
$$

The period in the optical system was controlled by changing the distance between the DOE and the optical pyramid. With the increasing distance, the separation distance between the four parallel sub-beams is increased, which results in a bigger interference angle and, in turn, a smaller interference pattern period. Further information about he used optical setup has been published elsewhere [17].

a)

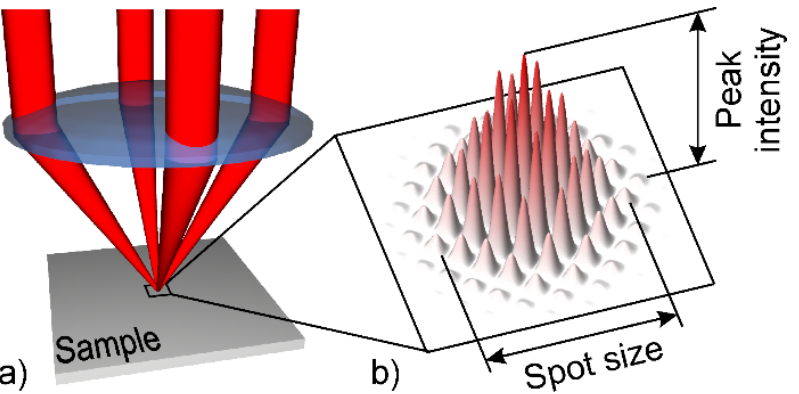

Fig. 1 Illustration of four beams focused and collected to a single point by a lens (a) and its resulting interference intensity profile (b).

\subsection{Topography analysis of the fabricated structures}

A scanning electron microscope (SEM, ZEISS Supra 40VP, Jena, Germany) was used to visualize the surface morphology of the laser-treated samples. The topography of the microstructures was also analyzed using a confocal microscope (Sensofar Sneox, Terrassa, Spain) equipped with a 150x magnification objective with a resolution of $170 \mathrm{~nm}$ and $2 \mathrm{~nm}$ in the lateral and vertical directions, respectively.

\section{Results and discussion}

The four-beam DLIP process was used to structure the steel sample surface. The experiments were implemented by varying the laser fluence between 0.4 and $2.4 \mathrm{~J} / \mathrm{cm}^{2}$ (which corresponds to the maximum laser output power), and the number of pulses per radiation between 1 and 25. The higher number of laser pulses was not analyzed in this paper because of the low beam pointing stability, which results in the lateral misalignment of the forthcoming pulses during longer exposures (more than 25 pulses).
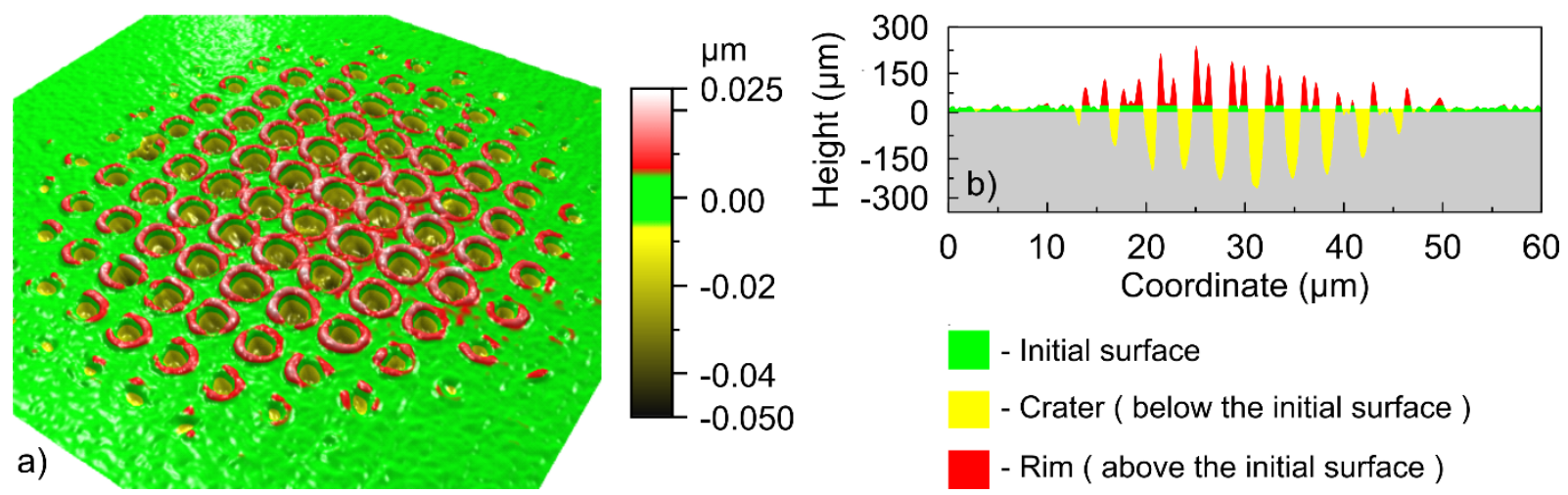

Fig. 2 The confocal image of the structure ablated by applying single pulse with $0.4 \mathrm{~J} / \mathrm{cm}^{2}$ fluence (a) and its cross-section (b). Green color denotes the surface that was not affected by the laser radiation, yellow - the surface inside the ablated crater, red - the material corresponding to the rims surrounding the craters. 
Each test was performed by irradiating the laser pulses at a single position. The same parameter variation was performed for two spatial structure periods, namely 1.8 and 3.5 $\mu \mathrm{m}$.

The confocal image of one exemplary structure ablated with a single pulse of $0.4 \mathrm{~J} / \mathrm{cm}^{2}$ is shown in Fig. 2a. The structure contains periodically distributed craters. Each crater is surrounded by a rim, which is higher than the unstructured surface. For a quantitative evaluation of the structure topography, three different regions can be recognized, which are (i) the unstructured surface, (ii) the produced crater, and (iii) the rims. These regions are colored in green, yellow, and red, respectively, as shown in Fig. $2 b$.

Each fabricated structure was characterized by measuring the depth of the crater $(\mathrm{Hc})$, the height of the rim $(\mathrm{Hr})$, and the total height of the structure $(\mathrm{Hstr}=\mathrm{Hc}+\mathrm{Hr})$. Only the central area of the ablated zone was analyzed, which corresponds to the peak fluence of the gaussian laser spot.

The analysis of the results is divided into two parts. The first part discusses the morphological characteristics of the structures formed with a single laser pulse and different laser fluences. The second part is dedicated to the structures produced with multiple pulses.

\subsection{Single-pulse laser ablation}

The height of the structures that were fabricated with a single laser pulse and different laser fluences was measured using confocal microscopy. Additionally, the depth of ablated craters and the heights of the rims surrounding the craters were also characterized. All measured data are plotted as a function of the used laser fluence for $3.5 \mu \mathrm{m}$ and $1.8 \mu \mathrm{m}$ period structures in Fig. 3a, and Fig. 3b, respectively.

The $3.5 \mu \mathrm{m}$ period structure height grows logarithmically when laser peak fluence is increasing from $0.4 \mathrm{~J} / \mathrm{cm}^{2}$ to $18.2 \mathrm{~J} / \mathrm{cm}^{2}$, reaching 60 and $270 \mathrm{~nm}$, respectively (1st stage, marked with green background in Fig. 3a). Such a trend is very common in the case of a single Gaussian beam ablation [18]. Suddenly, for high fluences up to $26 \mathrm{~J} / \mathrm{cm}^{2}$, the rate of the structure height growth significantly increased (2nd stage, blue background). However, the last used laser fluence $\left(27.4 \mathrm{~J} / \mathrm{cm}^{2}\right)$ resulted in a slight decrease of the structure height compared to the previous used lower fluence $\left(27 \mathrm{~J} / \mathrm{cm}^{2}\right)$ from 430 to $410 \mathrm{~nm}$ ( 3rd stage, red background).
The reason for such behavior can be found by looking into the rim height and crater depth plots, shown in Fig. 3a. It can be seen that at first, both the crater and the rim height increase with the laser fluence (1st stage). The increase of the rims' height is driven by the increasing amount of molten material that flows from the central part of the crater (see Fig. 4a). However, when the laser fluence reaches $18.2 \mathrm{~J} / \mathrm{cm}^{2}$, the height of the rims starts to grow faster, while the depth of craters continues the logarithmic growth, contributing to the rapid increase of the structure height.

To understand the reason for such sudden rim growth, a more detailed SEM and confocal microscopy analysis of the

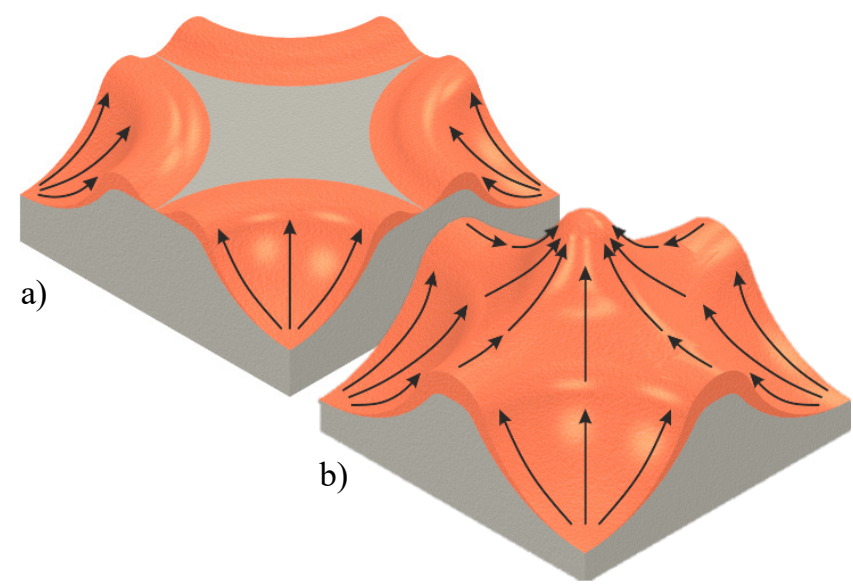

Fig. 4 The schematic representation of the molten material flow (represented by the black arrows) during the structure formation at stage 1 (a) and stage 2 (b).

structure topography was performed. The results are depicted in Fig. 5, where each image pair has a number, which corresponds to the specific point in the plots shown in Fig. 3. It can be seen that in the case of $3.5 \mu \mathrm{m}$ period, the sudden growth of the rims' height in the 2nd growth stage is related to the arising pillars in the areas between the craters (see, (3)-(5) in Fig. 5 for $\Lambda=3.5 \mu \mathrm{m}$ ). The growth of the pillars is driven by the molten material brought from the four adjacent craters (see Fig. 4b). The force that drives the molten material is induced by the recoil pressure formed during the rapid evaporation of the material during the laser ablation process. At fluences $\geq 18.2 \mathrm{~J} / \mathrm{cm}^{2}$, the amount of the molten material

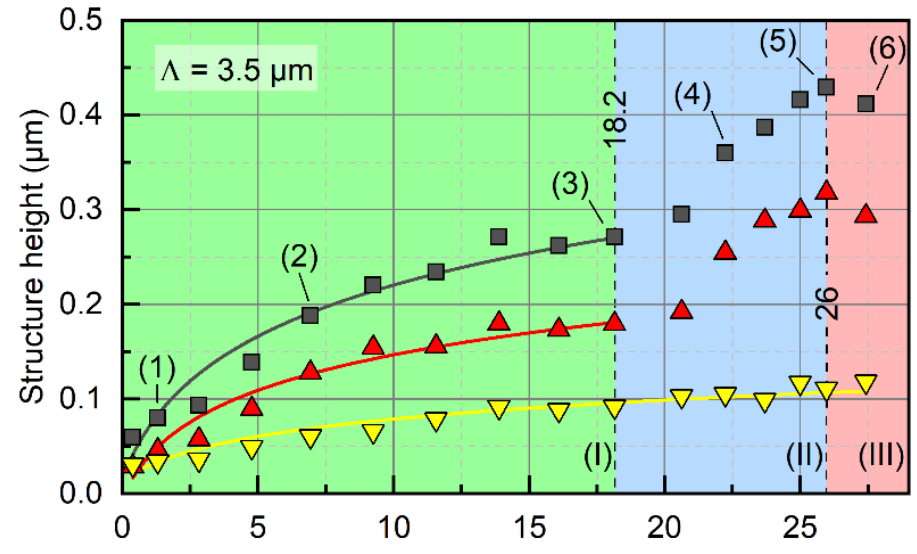

a)

Laser fluence $\left(\mathrm{J} / \mathrm{cm}^{2}\right)$

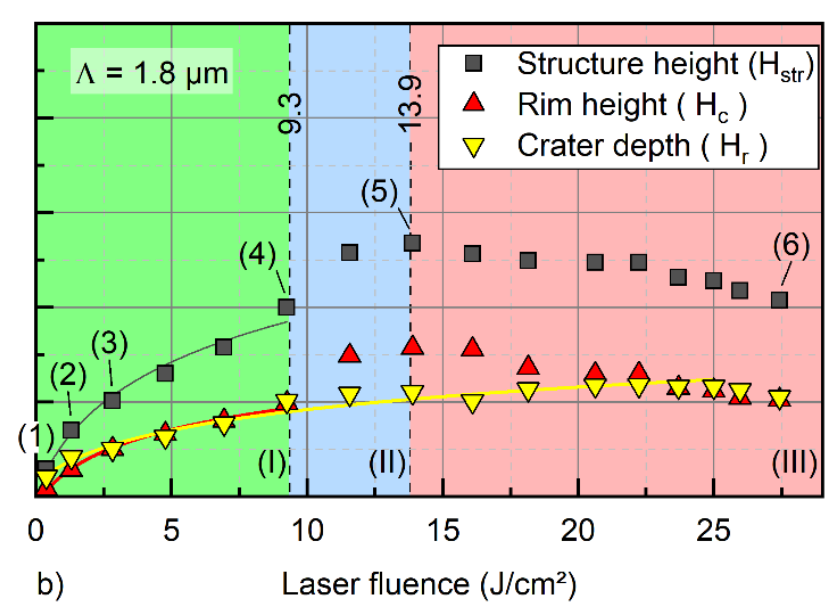

Fig. 3 The ablated craters depth, rims height and overall structure height dependence on applied laser fluence for structures dormed with $3.5 \mu \mathrm{m}$ (a) and $1.8 \mu \mathrm{m}$ interference pattern periods. 


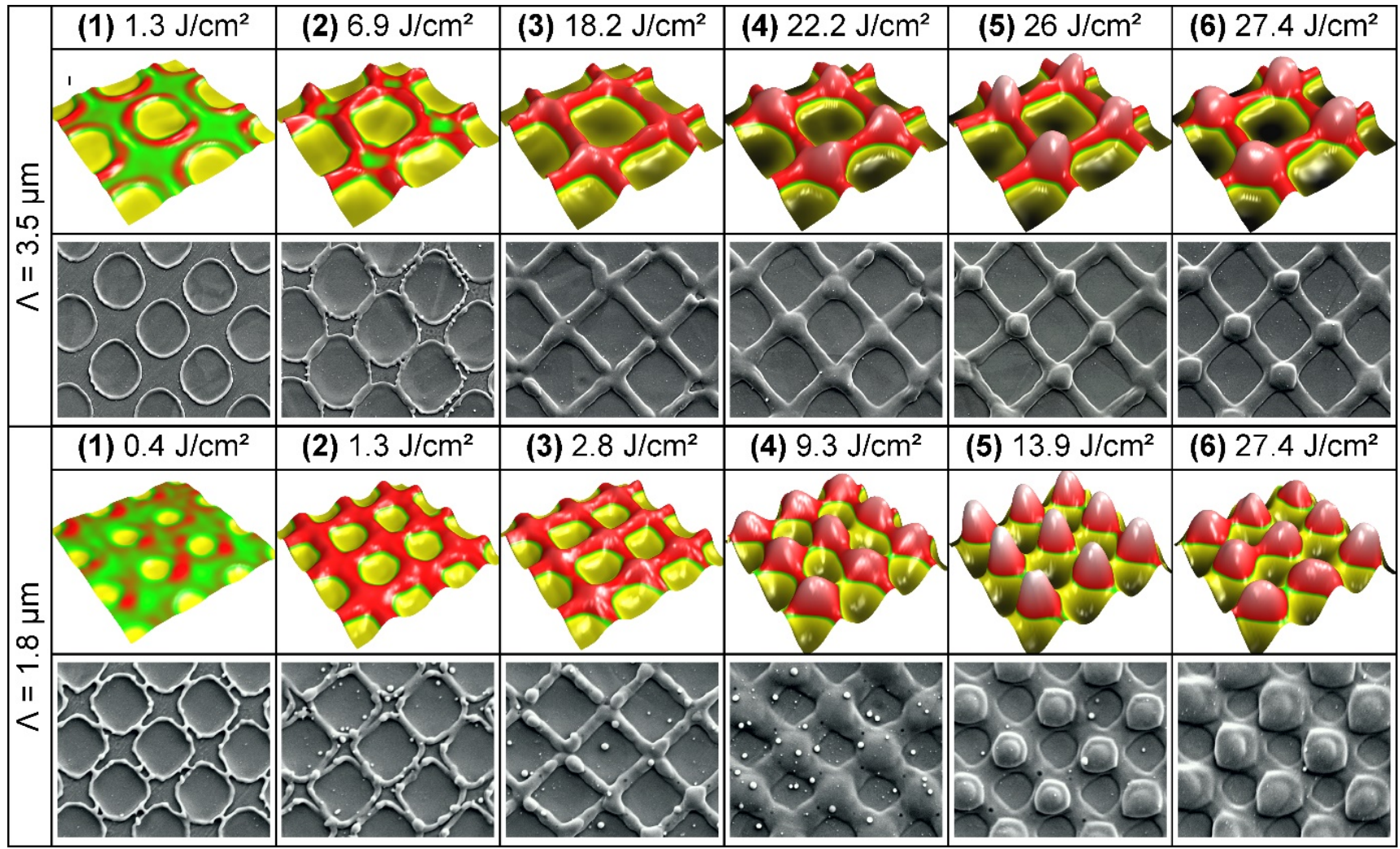

Fig. 5 The confocal microscopy (top rows) and SEM (bottom rows) micrographs of the structures fabricated using four beam DLIP with $3.5 \mu \mathrm{m}$ and $1.8 \mu \mathrm{m}$ interference pattern periods $(\boldsymbol{\Lambda})$.

becomes too large to fit in the area around the craters, and therefore it is driven vertically.

However, when the laser fluence becomes above $26 \mathrm{~J} / \mathrm{cm}^{2}$, the height of the formed pillar drops, resulting in a decrease of the overall structure height. Fig. 5 shows that when $\mathrm{F}=27.4 \mathrm{~J} / \mathrm{cm}^{2}$, the pillars are still formed, but their height is lower than in the case of $26 \mathrm{~J} / \mathrm{cm}^{2}$. The height drop can be explained since the molten material stays too long in a liquid state, providing enough time for the pillar to collapse due to its weight.

The structures with a smaller period of $1.8 \mu \mathrm{m}$ exhibit a similar growth trend (see Fig. 3b), except that in this case, the transition between the 1 st and the 2 nd stages is not so distinguishable as in case of $3.5 \mu \mathrm{m}$ period structures. This is because the distance that the molten material has to make in order to reach the pillar growth point is almost two times smaller. Therefore, the growth of the pillars starts at a relatively low fluence of $9.3 \mathrm{~J} / \mathrm{cm}^{2}$, without reaching the saturation of the structure height as it was in the case of $3.5 \mu \mathrm{m}$ period structures. Moreover, the growth of the pillars reaches its maximum also at lower fluence $\left(13.9 \mathrm{~J} / \mathrm{cm}^{2}\right)$, resulting in the much wider 3rd growth stage, where the structure height decreases with increasing laser fluence.

\subsection{Multiple pulse laser ablation}

In addition to single-pulse experiments with varied laser fluence, also the effect of the pulse number on the morphology of the produced DLIP structures using ps laser radiation was evaluated. The measured heights as a function of both laser fluence and a number of pulses are shown in Fig. 6a and Fig. $7 \mathrm{a}$, for $3.5 \mu \mathrm{m}$ and $1.8 \mu \mathrm{m}$ periods, respectively. The

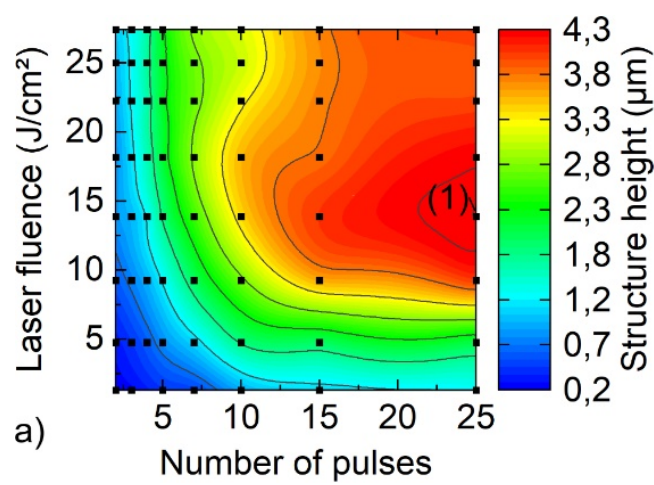

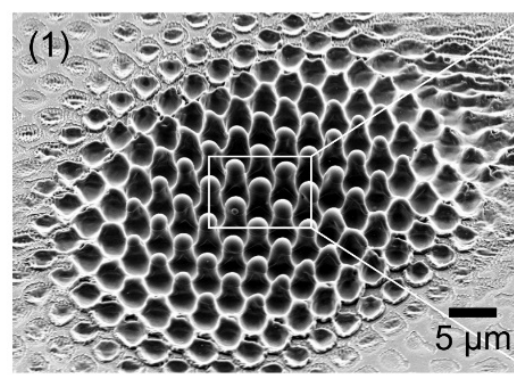

b)

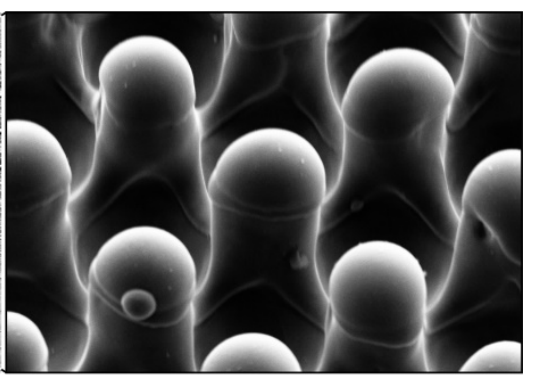

c)

Fig. 6 Structure height dependency for DLIP patterns with $3.5 \mu \mathrm{m}$ spatial period as a function of the laser fluence and number of applied laser pulses (a). SEM micrographs of the pattern with the maximal height faricated with $13.9 \mathrm{~J} / \mathrm{cm}^{2}$ laser fluence and 25 laser pulses (b). 
plots clearly show the optimal process parameters for structure fabrication with the highest structures.

In the case of a $3.5 \mu \mathrm{m}$ period and a low number of laser pulses (1-7), the structure height reaches its maximum at the highest used laser fluences $\left(\sim 25 \mathrm{~J} / \mathrm{cm}^{2}\right)$. Compared to the patterns obtained with only one laser pulse, these structures are approximately 10 times higher $(0.43 \mu \mathrm{m}$ vs. $4.3 \mu \mathrm{m})$.
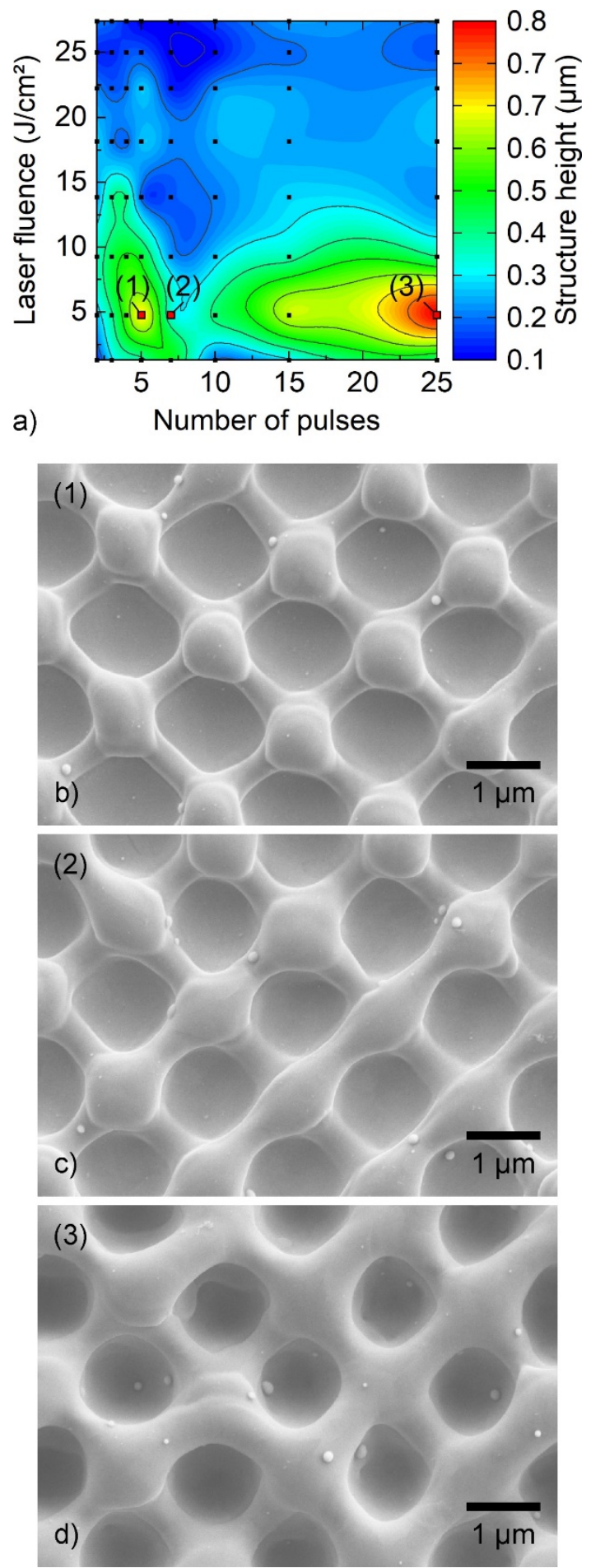

Fig. 7 Structure height dependency for DLIP patterns with $1.8 \mu \mathrm{m}$ spatial period as function of the laser fluence and number of applied laser pulses (a). SEM micrographs of the periodic structures produced at $4.8 \mathrm{~J} / \mathrm{cm}^{2}$ of laser fluence and 5 (b), 7 (c) and 25 (d) laser pulses, corresponding to the indications (1), (2) and (3) of figure (a), respectively.
Differently, with the increasing number of pulses, the structure height maximum shifts towards the lower laser fluences, reaching the overall highest structure at the maximal number of pulses used (25) and laser fluence of $13.9 \mathrm{~J} / \mathrm{cm}^{2}$. SEM micrographs of the structure formed using these parameters are shown in Fig. 6b, c. As can be seen, the pillar-like structure is formed in the central area of the Gaussian spot, which demonstrates that the pillars, formed with the first laser pulses, are continuously growing with each additional pulse. This can be explained, since each pulse brings the melted material from the crater area to the peak of the pillar, increasing its height.

A very different result was observed for the case of $1.8 \mu \mathrm{m}$ structures (see structure height map in Fig. 7a). Here the maximal structure heights correspond to the area of low laser fluences for all used number of pulses. Moreover, instead of having a single region with the highest patterns (as in $3.5 \mu \mathrm{m}$ structures), the height-map shows two characteristic areas. Both periodic structures are formed at a laser fluence of $4.8 \mathrm{~J} / \mathrm{cm}^{2}$. These peaks are reached with 5 and 25 pulses (see (1) and (3) in Fig. 7a). Between these process parameters, the structure height decreases, reaching a minimum when 7 laser pulses are used (see (2) in Fig. 7a). The SEM micrographs of the structures formed with the process parameters corresponding to the mentioned three regions are shown in Fig. 7b-d. It can be seen that at the first peak (corresponding to 5 laser pulses), a pillar-like geometry with very-well defined shape characterizes the produced surface topography (see Fig. 7b). Then, by increasing the number of pulses to 7, because of the excess of the additional molten material, the formed pillars collapse and spread around the craters (Fig. 7c). The further increase of the number of laser pulses provides an additional delivery of the melted material on top of the previously formed rims resulting in the growth of the average structure height but producing very-well defined craters. (Fig. 7d). In consequence, the variation of the process parameters does not only allow to control the height of the structures but also to change the pattern morphology from pillar-like to crater-like geometries.

\section{Conclusions}

The rigorous analysis of the structures formed with fourbeam DLIP of a steel surface showed a significant role of the molten material dynamics in the final structure topographical parameters. Different behaviors were observed depending on the used spatial period, laser fluences, and a number of pulses.

For the steel samples treated with only one laser pulse, at low laser fluences, periodically distributed craters were fabricated, at the positions corresponding to the interference maxima of the laser intensity distribution. However, when the laser fluence surpassed specific thresholds $\left(18.2 \mathrm{~J} / \mathrm{cm}^{2}\right.$ and $9.3 \mathrm{~J} / \mathrm{cm}^{2}$, for $3.5 \mu \mathrm{m}$ and $1.8 \mu \mathrm{m}$ period structures, respectively), pillars are formed in between the craters. The primary mechanism of the pillar formation was found to be the material flow from the area of the craters. It was also discovered that the pillars form at lower laser fluences when smaller periods are applied $(1.8 \mu \mathrm{m})$. This effect was explained due to the smaller travel distance of the molten material for the shorter periods.

The experiments with multiple laser pulses showed that the height of the produced structures corresponding to the 
$3.5 \mu \mathrm{m}$ period could be increased about ten times (from $0.43 \mu \mathrm{m}$ to $4.3 \mu \mathrm{m}$ ) when raising the pulse number from 1 to 25 . In this case, also a structured geometry consisting of pillars was obtained, which indicates that each forthcoming laser pulse brings additional material on top of the previously formed pillar, increasing its height. However, in the case of a smaller spatial period $(1.8 \mu \mathrm{m})$, the formation of pillars was only possible at a low number of laser pulses (5). A further increase of the pulse number resulted in the collapse of the pillars and the molten material distributes around the craters. In this case, by applying 25 laser pulses, the structure height was increased only 3 times: from $0.27 \mu \mathrm{m}$ (with single laser pulse) to $0.82 \mu \mathrm{m}$.

\section{Acknowledgments}

This project was funded by the European Regional Development Fund (ERDF) and co-financed under taxation on the basis of the budget adopted by the members of the Saxon State Parliament.

\section{References}

[1] Y. Nakata: Adv. Opt. Technol., 5, (2016) 29.

[2] V. Lang, A. Rank, and A.F. Lasagni: Adv. Eng. Mater., 19, (2017) 1700126.

[3] S. Milles, B. Voisiat, M. Nitschke, and A.F. Lasagni: J. Mater. Process. Technol., 270, (2019) 142.

[4] S. Milles, M. Soldera, B. Voisiat, and A.F. Lasagni: Sci. Rep., 9, (2019) 13944.

[5] A. Peter, A.H.A. Lutey, S. Faas, L. Romoli, V. Onuseit, and T. Graf: Opt. Laser Technol., 123,
(2020) 105954.

[6] P. Graus, T.B. Möller, P. Leiderer, J. Boneberg, and N.I. Polushkin: Opto-Electronic Adv., 3, (2020) 190027.

[7] B. Voisiat, W. Wang, M. Holzhey, and A.F. Lasagni: Sci. Rep., 9, (2019) 7801.

[8] M. D’Alessandria, A. Lasagni, and F. Mücklich: Appl. Surf. Sci., 255, (2008) 3210.

[9] M. Gedvilas, S. Indrišiunas, B. Voisiat, E. Stankevičius, A. Selskis, and G. Račiukaitis: Phys. Chem. Chem. Phys., 20, (2018) 12166.

[10] S.A. Khairallah, A.T. Anderson, A. Rubenchik, and W.E. King: Acta Mater., 108, (2016) 36.

[11] A. Ben-Yakar, A. Harkin, J. Ashmore, R.L. Byer, and H.A. Stone: J. Phys. D. Appl. Phys., 40, (2007) 1447.

[12] Y. Nakata, N. Miyanaga, K. Momoo, and T. Hiromoto: Appl. Surf. Sci., 274, (2013) 27.

[13] V. Lang, B. Voisiat, and F.A. Lasagni: Materials, 12, (2019) 1484.

[14] B. Voisiat, C. Zwahr, and A.F. Lasagni: Appl. Surf. Sci., 471, (2019) 1065.

[15] B. Voisiat, M. Gedvilas, S. Indrišiūnas, and G. Račiukaitis: J. Laser Micro/Nanoengineering, 6, (2011) 185.

[16] S. Indrišiūnas, B. Voisiat, M. Gedvilas, and G. Račiukaitis: Proc. LPM2017, (2017) \#17-60.

[17] M. El-Khoury, S. Alamri, B. Voisiat, T. Kunze, and A.F. Lasagni: Mater. Lett., 258, (2020) 126743.

[18] A. Žemaitis, M. Gaidys, M. Brikas, P. Gečys, and G. Račiukaitis: Sci. Rep., 8, (2018) 17376.

(Received: June 19, 2020, Accepted: February 1, 2021) 